\title{
Analytical Approach for Making Multilayer and Inhomogeneous Structures Invisible Based on Mantle Cloak
}

\author{
${ }^{1}$ E. G. Mizuji, ${ }^{2}$ A. Abdolali, and ${ }^{3}$ F. Aghamohamadi \\ ${ }^{1-3}$ Department of Electrical Engineering, Iran University of Science and Technology, \\ e-mail:ensiyeh.ghasemi@ee.iust.ac.ir
}

\begin{abstract}
We investigate an analytical approach based on transmission line model to design mantle cloak for multilayer and/or inhomogeneous dielectric objects. Our proposed model is verified with different numerical results. The results matched well with each other. Also, the required impedance for making an inhomogeneous material invisible is calculated analytically. Finally, some complex structures are implemented using CST simulator and suitable performance is reached. So, it is proved that mantle cloak is applicable for making complex inhomogeneous materials invisible.
\end{abstract}

Index Terms - Mantle cloak, Inhomogeneous material, Frequency selective surface (FSS), Scattering cancellation.

\section{INTRODUCTION}

The idea of making an object invisible based on transformation optics was first introduced by Pendry [1]. He acclaimed that the direction of incident waves can be controlled by means of coating metamaterials [1-3]. Many efforts have been devoted to design various types of cloaks so far [4-7]. However, bulk configuration, heavy weight and narrow bandwidth are serious issues in many proposed platforms.

Plasmonic cloaks [8-9] and mantle cloaks [10] also have been studied based on scattering cancelation, and as a result FSSs is implemented to achieve cloaking at RF spectrum [10] while at terahertz graphene is utilized for making object invisible [11]. In comparison to bulk metamaterial cloaks, mantle cloak benefits from low profile, low volume, low cost and feasibility of designing and manufacturing. The main drawback of mantle cloaks is their narrow bandwidth. As an approach to broaden the bandwidth, multilayer FSS is deployed [12-13]. Experimental results have also proven good performance of mantle cloak [14-15]. This type of cloak has been studied under illumination of plane wave [16] or line source [17].

In [18], both cylindrical PEC (Perfect electric conductor) and dielectric objects covered with FSS are studied under illumination of both TE (transverse electric) and TM (transverse magnetic) waves. Some applications in acoustic cloaking are reported in [19] for mantle cloak. Moreover, mantle cloak is utilized to minimize the scattering of antennas [20] and some applications for co site radio frequency antennas are introduced in [21]. Additionally, mantle cloak found a lot of applications in 
antennas [22-23]. It has been successfully employed for making anisotropic objects invisible [24]. Also, the metasurface anisotropy for achieving low profile cloak has been exploited in [25].

In this paper some close-form equations have been presented in order to analyze multilayer structures. The analytical equations required to calculate the cloak impedance and the separation distance between the object and the mantle cloak, are obtained using transmission line method. To verify the suggested equations some complex structures are implemented in ADS and CST simulators. Simulation results show good agreement with analytical solutions. Furthermore, an inhomogeneous material is considered and solved by dividing it into thin homogenous layers by means of abovementioned method. The required impedance for making this object invisible is calculated using the close form equation.

\section{DEVELOPMENT OF THE PROPOSED MANTLE CLOAK FOR MULTILAYER SLABS}

In this section a multilayer structure covered by a mantle cloak layer based on transmission line method has been modeled and shown in Figure 1a, 1b and 1c. As can be seen there is a multilayer object covered by a frequency selective surface (FSS) and there is a gap between the object and the FSS as it is necessary for designing a mantle cloak. all the layers are assumed to be homogenous and the layers are electrically thin $(\beta \mathrm{d}<<\lambda)$ and it is supposed all the structures are under illumination of plane wave with normal incident angle. In order to deliver analytical equations for cloaking multilayer structures at first step a two-layer structure is studied using transmission line model, equating $S_{11}$ to zero (figure 1-b). Therefore, the equations 1 and 2 are found for deriving $X s$ and $d . X s$ and $d$ have been defined as the required impedance for making the structure invisible and the optimum distance between $X s$ and the structure to achieve maximum cloaking, respectively.

$$
\begin{gathered}
X_{s}=\frac{\eta_{0}^{2} \eta_{1} \eta_{2}}{\eta_{0}^{2}\left(\eta_{2} \phi_{1}+\eta_{1} \phi_{2}\right)-\eta_{1} \eta_{2}\left(\eta_{1} \phi_{1}+\eta_{2} \phi_{2}\right)} \\
\phi_{0}=\frac{X_{s} \eta_{0} \phi_{1} \phi_{2}\left(\eta_{2}^{2}-\eta_{1}^{2}\right)+\eta_{0} \eta_{1} \eta_{2}\left(\eta_{1} \phi_{1}+\eta_{2} \phi_{2}\right)}{X_{s}\left(\eta_{1} \eta_{2}\left(\eta_{1} \phi_{1}+\eta_{2} \phi_{2}\right)+\eta_{0} \eta_{2}^{2} \phi_{1} \phi_{2}\right)+\eta_{0}^{2}\left(\eta_{1} \phi_{1}+\eta_{2} \phi_{2}\right)-\eta_{0}^{2} \eta_{1} \eta_{2}+\eta_{0}^{2} \eta_{1}^{2} \phi_{1} \phi_{2}} \\
\phi_{0}=\beta_{0} d_{0}
\end{gathered}
$$

These equations can be extended to 4 and 5 for multilayer structures.

$$
\begin{gathered}
X_{s}=\frac{\eta_{0}^{2} \eta_{1} \eta_{2} \ldots . . \eta_{i}}{\eta_{0}^{2} \sum_{n=1}^{i}\left(\eta_{1} \eta_{2} . . \eta_{m-1} \eta_{m+1} \ldots . \eta_{n}\right) \phi_{m}-\eta_{1} \eta_{2} \ldots \eta_{i} \sum_{n=1}^{i} \eta_{n} \phi_{n}} \\
\phi_{0}=\frac{X_{s} \eta_{0} \phi_{1} \phi_{2}\left(\eta_{2}^{2}-\eta_{1}^{2}\right)+\eta_{0} \eta_{1} \eta_{2}\left(\eta_{1} \phi_{1}+\eta_{2} \phi_{2}\right)}{X_{s}\left(\eta_{1} \eta_{2}\left(\eta_{1} \phi_{1}+\eta_{2} \phi_{2}\right)+\eta_{0} \eta_{2}^{2} \phi_{1} \phi_{2}\right)+\eta_{0}^{2}\left(\eta_{1} \phi_{1}+\eta_{2} \phi_{2}\right)-\eta_{0}^{2} \eta_{1} \eta_{2}+\eta_{0}^{2} \eta_{1}^{2} \phi_{1} \phi_{2}} \\
\phi_{i}=\beta_{i} d_{i}
\end{gathered}
$$

Where $\lambda_{\mathrm{i}}, \eta_{\mathrm{i}}$ and $\beta_{\mathrm{i}}$ are wavelength, impedance and wave number of ith layer, respectively. In these equations the high order sentences of $\phi_{\mathrm{i}}$ are ignored. Equations 1 and 2 can be generalized for an iBrazilian Microwave and Optoelectronics Society-SBMO received 26 Dec 2017; for review 04 Feb 2018; accepted 15 March 2018 
layer structure. By writing the transfer matrix of each layer then deriving the total impedance of the structure $\mathrm{z}_{\text {total }}$, Equations 4 to 6 can be obtained. The transfer matrix and $\mathrm{z}_{\text {total }}$ are expressed as in equations 7 and 8 , respectively. Xs and $\phi_{0}$ are obtained by equating the reflection $\Gamma$ of the structure to zero. This approach has been used in relevant studies [26] to solve scattering problems.

$$
\begin{aligned}
& {[T]=\left[\begin{array}{cc}
\cos \left(\phi_{i}\right) & j \cdot \eta_{i} \cdot \sin \left(\phi_{i}\right) \\
j \cdot \sin \left(\phi_{i}\right) / \eta_{i} & \cos \left(\phi_{i}\right)
\end{array}\right]} \\
& z_{\text {total }}=\frac{A \eta_{0}+B}{C \eta_{0}+D}
\end{aligned}
$$

In the transmission line model, the frequency selective surface is modeled by the impedance of $z_{d}$. In order to validate the above-mentioned equations some multilayer structures are explored. The scattering problem is solved by two different methods. At first $\mathrm{S}$ parameters are calculated with use of transmission line model (Fig.1.b) and equations 9, 10 and 11.

$$
\begin{gathered}
S_{11}=|\Gamma|^{2} \\
S_{21}=1-|\Gamma|^{2} \\
\Gamma=\left|\frac{\eta_{0}-z_{\text {total }}}{\eta_{0}+z_{\text {total }}}\right|
\end{gathered}
$$

Then, the transmission and reflection coefficients are found analytically by writing the electric and magnetic fields in each layer and forcing the boundary conditions. These fields are shown in figure 1.c. The boundary condition for the tangential magnetic field on the FSS surface is specified by equation 11 and the tangential electric field is continuous.

$$
\left.E_{t}\right|_{z=0^{+}}=\left.E_{t}\right|_{z=0^{-}}=Z_{s}\left(\left.H_{t}\right|_{z=0^{+}}-\left.H_{t}\right|_{z=0^{-}}\right)
$$

In all other boundaries tangential components of both electric and magnetic fields are continuous.

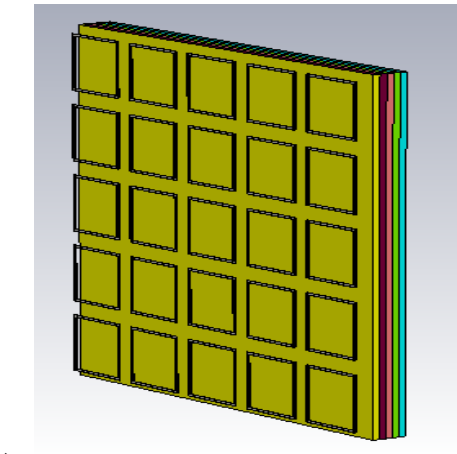

a)

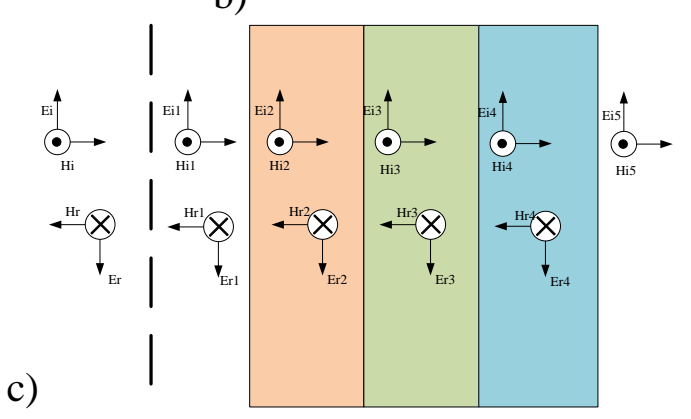

Fig. 1. a) The multilayer planar geometry covered with FSS cloak. b) The equivalent transmission line model. c) the electric and magnetic fields in all the layers. The FSS is shown with the dashed line.

Brazilian Microwave and Optoelectronics Society-SBMO received 26 Dec 2017; for review 04 Feb 2018; accepted 15 March 2018 
Consider a two layered material with the same physical thickness of $\lambda / 100$ which satisfies the condition of $\beta \mathrm{d}<<\lambda_{0}$. The electric constant of $\varepsilon_{\mathrm{r}}$ for the first and second layers are 12 and 8 , respectively. All the values for wavelength and permittivity in this paper are chosen merely for illustrating proposed approach. A plane wave excites whole structure. From equation 4 the required $Z_{\mathrm{s}}$ and the separation distance are calculated and chosen equal to $\mathrm{j} 333 \mathrm{ohm}$ and $\lambda / 150$, respectively. Figure 2 shows the $\mathrm{S}$ parameters achieved with both methods for the single dielectric and cloak object brought using MATLAB. As it is clear from figure, most of the wave is transmitted ( $\mathrm{S} 21$ is near $0 \mathrm{~dB}$ ) and reflection is minimized (S11 improved more than $10 \mathrm{~dB}$ in comparison to the uncloaked slab) at the designed frequency of $\mathrm{f}_{0}$. As a result, the scattering is drastically suppressed. There is a little shift in the center frequency due to the mentioned approximations.

To verify generality of the attained equations, a five layer material is considered. In this structure the total thickness is $\lambda / 50$ and all the layers have the same physical thickness. The dielectric constant of layers are 7,13,10,14 and 16, respectively. The $S$ parameters of the cloaked object and single dielectric are plotted in figure 3-a. In this figure $S$ parameters are calculated by the methods mentioned perviously and ADS simulator. All the results matched well with each other. The impelemented ADS circuit is drawn in figure 3-b. The technique mentioned in [27] is employed to bring ADS S parameters. In this model the dielectric layers are modeled by the transmission line and FSS layer is exhibited as an inductor. Cloaking properties have been reached by placing an inductive impedance equal to $\mathrm{j} 272 \mathrm{ohm}$ with spacing distance of $\lambda / 150$. Analytical and ADS S parameters matched well with each other.

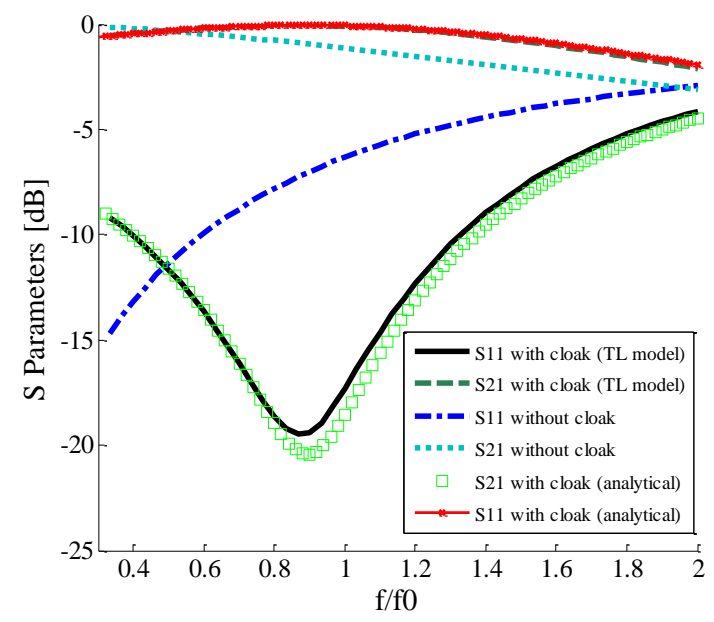

Fig.2. The S parameters for twolayer structure with and without cloak.

The equations are explored for more structures to calculate the values of $\varepsilon_{\mathrm{r}}$. The arrangment of the layers are not important and the obtained equations have generality. Different structures are considered and have been shown in table I. In this table, $d$ is the distance of FSS from object and $Z$ is the required impedance. 
a)

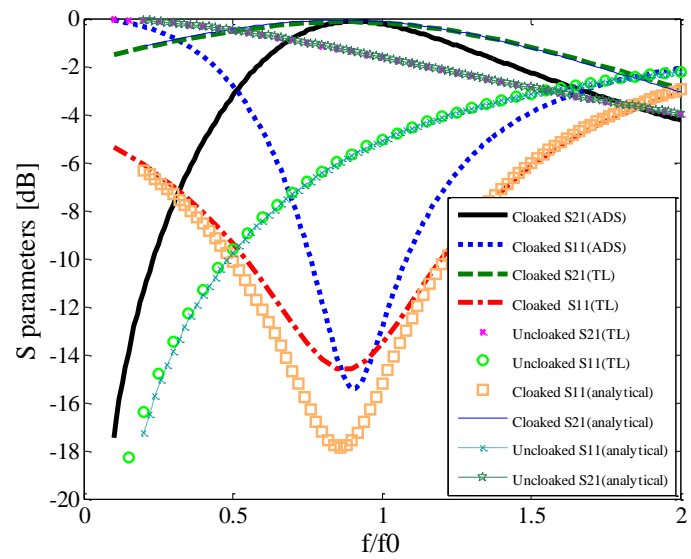

b)

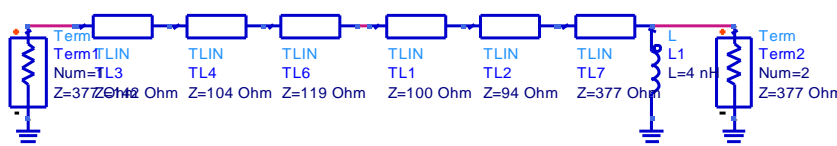

Fig.3. a) The $\mathrm{S}$ parameters for 5 layer structure with and without cloak. b) ADS model for this structure.

$\mathrm{S}$ parameters of these strucures obtained from analytical equations are shown in figure 4 . These values are chosen randomly. In all structures all of the layers have the same physical thickness and the total thickness of all structures is $\lambda / 50$. The S21 and S11 for all of the structures show good performance of suggested equations.

TABLE I. THE PROPERTIES FOR SOME DIFFERENT STRUCTURES STUDIED IN FIG.4

\begin{tabular}{lllllll}
\hline $\mathbf{1}_{\text {st }}$ layer $\boldsymbol{\varepsilon}_{\mathbf{r}}$ & $\mathbf{2}_{\text {nd }}$ layer $\boldsymbol{\varepsilon}_{\mathbf{r}}$ & $\mathbf{3}_{\mathbf{r d}}$ layer $\boldsymbol{\varepsilon}_{\mathbf{r}}$ & $\mathbf{4}_{\text {th }}$ layer $\boldsymbol{\varepsilon}_{\mathbf{r}}$ & $\mathbf{5}_{\text {th }}$ layer $\boldsymbol{\varepsilon}_{\mathbf{r}}$ & $\mathbf{d}$ & $\mathbf{Z}$ \\
\hline 20 & 5 & 12 & 11 & 9 & $\lambda / 90$ & $\mathrm{j} 288$ \\
6 & 15 & 18 & 13 & 11 & $\lambda / 90$ & $\mathrm{j} 258$ \\
7 & 13 & 10 & 14 & 16 & $\lambda / 90$ & $\mathrm{j} 272$ \\
\hline
\end{tabular}

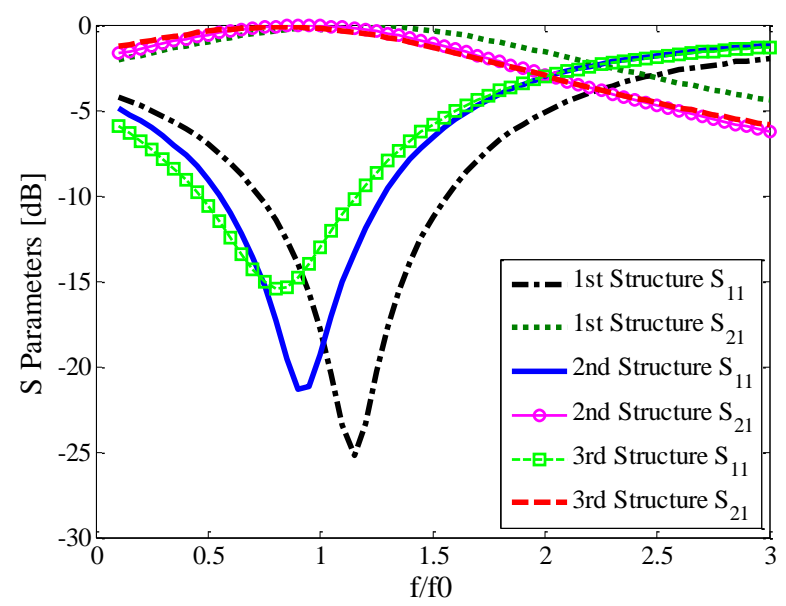

Fig.4. The S parameters for different 5 layer structures.

\section{STUDY OF SOME INHOMOGENEOUS SLABS}

Most of the materials in the nature are inhomogeneous. One method for solving scattering problem in an inhomogeneous material is dividing the material into small sub-layers and considering these 
layers as homogeneous material, so wave equations in a homogeneous material can be used. Figure 5 depicts this technique.

Consider two inhomogeneous slabs with the dielectric constants of $\varepsilon_{r}=4 * 10^{4} z^{2} / \lambda^{2}+1$ and $\varepsilon_{r}=54 \log (50 * z / \lambda+1)+1$ with the thickness of $\lambda / 50$. These dielectric constants are chosen randomly and since they are common profiles in the nature. Additionally, square profile is the simplest inhomogeneous profile. $\varepsilon_{\mathrm{r}}$ of the first slab is increased with increasing of the thickness unlike the other one. To solve scattering problem for these structures they have been divided it into small sublayers. It has been started with dividing the slab into the two homogeneous parts and then it continued with $\mathrm{n}$ sub-layer. While the calculated $\mathrm{z}_{\mathrm{x}}$ for the slab with $\mathrm{n}$ sub-layers shows little error in comparison to the slab divided into $\mathrm{n}+1$ sub-layers. Equation 4 is utilized for calculating the $\mathrm{z}_{\mathrm{x}}$.

As it is shown in figure 5, after dividing inhomogeneous slabs into small sub-layers, $\varepsilon_{\mathrm{r}}$ in each sublayer is considered equal to the average $\varepsilon_{\mathrm{r}}$ of the beginning and ending points of that sub-layer. This method makes little error. For $n=7$ the approximated $\varepsilon_{\mathrm{r}}$ has good agreement with the original $\varepsilon_{\mathrm{r}}$ (figure 5) since having more sub-layer leads to have more accurate response. As the distance between object and FSS has a little effect on the response it is assumed to be $\lambda / 61$ in all the stages. Table II shows the calculated $z_{x}$ in each stage. Figure 6 demonstrates the computed impedance versus the number of the sub-layers for both profiles. These curves prove the total impedances are j562 ohm and j331 ohm for square and logarithmic profiles, respectively. The calculated impedances for $n=7$ are $j 557$ and $j 331.72$ with negligible error of $0.8 \%$ and $0.2 \%$, respectively. Lower error of the approximated impedance for the second profile is due to its smoother variation versus thickness. It means the suggested square profile is closer to linear in compare with the suggested logarithm profile.

The error is estimated with subtracting the calculated impedance in each stage from the final value and dividing the attained value into final value. The final impedance value is achieved when $n$ is going toward infinity. Equation 13 describes the error:

$$
\text { error }_{i}=\left|\frac{Z_{\text {final }}-Z i}{Z_{\text {final }}}\right|
$$

TABLE II. THE CALCULATED IMPEDANCE FOR CLOAKING INHOMOGENEOUS SLAB VERSUS NUMBER OF SUB-LAYER.

\begin{tabular}{ccccc}
\hline Number of slides & $\begin{array}{c}\text { Calculated impedance } \\
\text { 1st profile }\end{array}$ & error & $\begin{array}{c}\text { Calculated impedance } \\
\text { 2nd profile }\end{array}$ & error \\
\hline 2 & $\mathrm{j} 499$ & $11 \%$ & $\mathrm{j} 340.040$ & $2 \%$ \\
3 & $\mathrm{j} 532$ & $5.3 \%$ & $\mathrm{j} 334.98$ & $1 \%$ \\
4 & $\mathrm{j} 545$ & $3 \%$ & $\mathrm{j} 333.23$ & $0.6 \%$ \\
5 & $\mathrm{j} 551$ & $1.9 \%$ & $\mathrm{j} 332.43$ & $0.4 \%$ \\
6 & $\mathrm{j} 554$ & $1.4 \%$ & $\mathrm{j} 331.99$ & $0.2 \%$ \\
7 & $\mathrm{j} 557$ & $0.8 \%$ & $\mathrm{j} 331.72$ & $0.2 \%$ \\
10 & $\mathrm{j} 559$ & $0.5 \%$ & $\mathrm{j} 331.35$ & $0.1 \%$ \\
20 & $\mathrm{j} 561$ & $0.1 \%$ & $\mathrm{j} 331.08$ & $0 \%$ \\
80 & $\mathrm{j} 562$ & $0 \%$ & $\mathrm{j} 331.005$ & $0 \%$ \\
100 & $\mathrm{j} 562$ & $0 \%$ & $\mathrm{j} 331.002$ & $0 \%$ \\
\hline
\end{tabular}



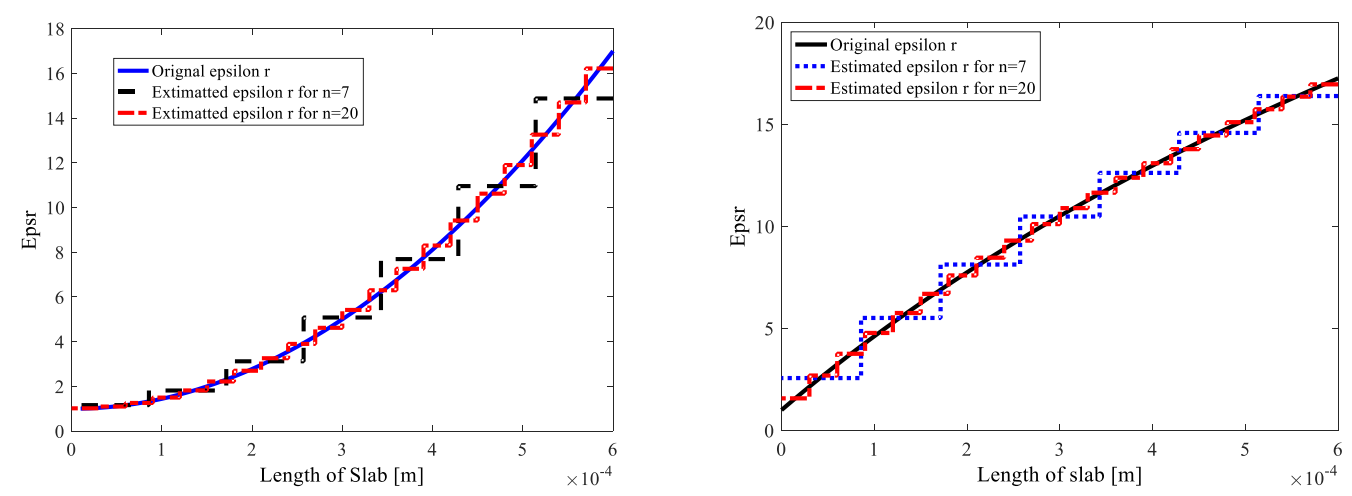

Fig.5. The estimated $\varepsilon_{\mathrm{r}}$ versus the length of slab.

The reflection and transmission coefficients are shown for 3-7 sub-layers in figure 7. The required inductive impedances for making the material invisible in each stage are brought in table II. The calculated impedances for $\mathrm{n}=7$ show $0.8 \%$ and $0.2 \%$ error for first and second profiles, respectively. So, the $\mathrm{S}$ parameters obtained for $\mathrm{n}=7$ can be considered as the $\mathrm{S}$ parameters for the inhomogeneous slab. As can be seen in figure 7 for $n>4 S$ parameters are almost similar. As it is expected from table II. The S parameters of logarithmic profile divided into 3 sub-layers have lower error than square profile due to its smoother changes versus thickness. As it is mentioned before the suggested logarithmic profile is closer to linear than the suggested square profile.

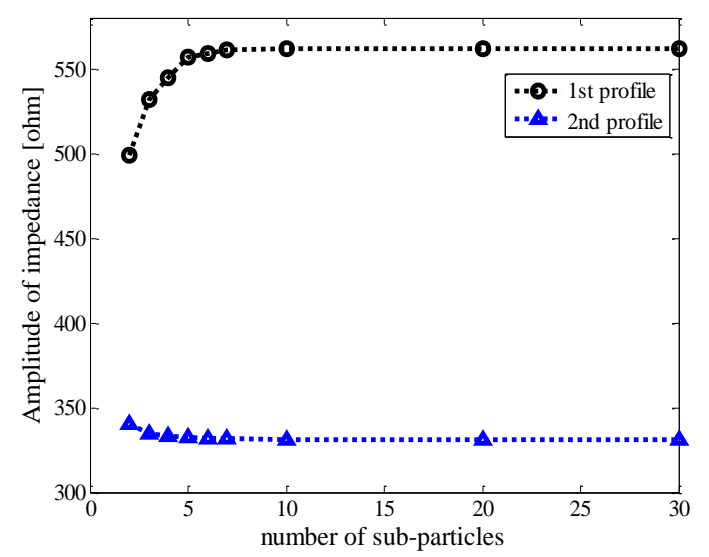

Fig.6. The impedance required for cloaking the inhomogeneous slab versus number of sub-particles.
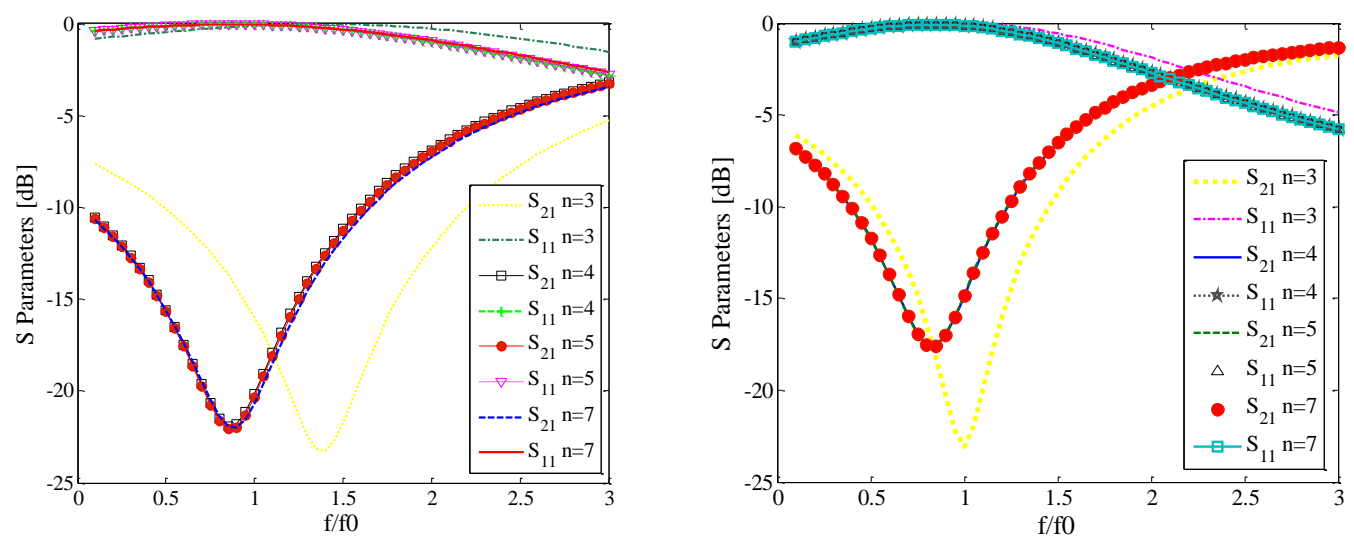

Fig.7. The S parameters for the inhomogeneous cloaked slab which is approximated with 3 to 7 homogeneous sub-layers. a) square profile. b) logarithmic profile.

Brazilian Microwave and Optoelectronics Society-SBMO received 26 Dec 2017; for review 04 Feb 2018; accepted 15 March 2018 Brazilian Society of Electromagnetism-SBMag 


\section{STUDY OF SOME INHOMOGENEOUS CYLINDERS}

The ability of mantle cloak to make 2D multilayered structures invisible has been presented in this section. Consider a multilayered cylinder under illumination of TM electromagnetic wave (Fig.8). The FSS is shown using dash line and placed with a little space from the object. Different materials are shown with different colors.

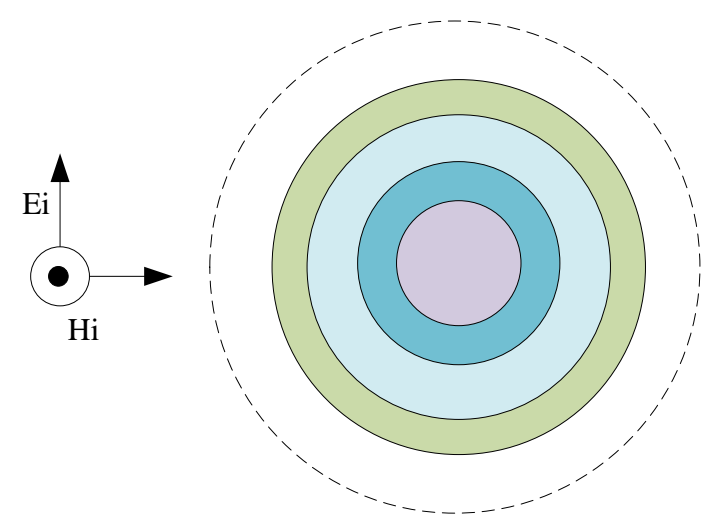

Fig.8. The schematic of multilayer cylinder under illumination of TM wave.

The electric fields in each layer are considered as:

$$
\begin{aligned}
& E_{z}^{i n c}=E_{0} \sum_{n=-\infty}^{\infty}(-j)^{n} J_{n}\left(k_{N+1} \rho\right) e^{j n \phi} \\
& E_{z}^{s}=E_{0} \sum_{n=-\infty}^{\infty}(-j)^{n} A_{n} H_{n}^{(2)}\left(k_{N+1} \rho\right) e^{j n \phi} \\
& E_{z}^{1}=E_{0} \sum_{n=-\infty}^{\infty}(-j)^{n} D_{n}^{1} J_{n}\left(k_{d} \rho\right) e^{j n \phi} \quad \rho<r_{1} \\
& E_{z}^{i}=E_{0} \sum_{n=-\infty}^{\infty}(-j)^{n}\left[C_{n}^{i+1} H_{n}^{(1)}\left(k_{0} \rho\right)+D_{n}^{i+1} H_{n}^{(2)}\left(k_{0} \rho\right)\right] e^{j n \phi} \quad r_{i}<\rho<r_{i+1} \quad i=1 \ldots \ldots . . n
\end{aligned}
$$

In above equations $\mathrm{E}^{\mathrm{inc}}$ and $\mathrm{E}^{\mathrm{s}}$ are incident electric field and scattered electric field, respectively. The electric field in ith layer is demonstrated with $\mathrm{E}^{\mathrm{i}}$. The electric field in the inner cylinder is described with $\mathrm{E}^{1}$. Also magnetic fields can be easily obtained from below equation:

$$
H_{\phi}=\frac{1}{j \omega \mu} \frac{\partial E_{z}}{\partial \rho}
$$

The unknown coefficients are computed using boundary conditions which are explained in [10]. Some different multilayered profiles have been chosen in this study. The specifications of these profiles are shown in table III. For these profiles the $z_{\mathrm{s}}$ is computed using equation 19 . The total $\varepsilon_{\mathrm{r}}$ is considered as the average electric constants of all layers.

$$
z_{s}=\frac{2}{\operatorname{wra\varepsilon }_{0}\left(\frac{\sum_{n=1}^{N} \varepsilon_{r(i)}}{N}+1\right)}
$$


Where $\varepsilon_{\mathrm{r}(\mathrm{i})}$ is the electric constant of the ith layer.

TABLE III. THE SPECIFICATION OF DIFFERENT INHOMOGENEOUS PROFILES.

\begin{tabular}{lllllll}
\hline $\mathbf{1}_{\mathbf{s t}}$ layer $\boldsymbol{\varepsilon}_{\mathbf{r}}$ & $\mathbf{2}_{\text {nd }}$ layer $\boldsymbol{\varepsilon}_{\mathbf{r}}$ & $\mathbf{3}_{\text {rd }}$ layer $\boldsymbol{\varepsilon}_{\mathbf{r}}$ & $\mathbf{4}_{\text {th }}$ layer $\boldsymbol{\varepsilon}_{\mathbf{r}}$ & $\mathbf{5}_{\text {th }}$ layer $\boldsymbol{\varepsilon}_{\mathbf{r}}$ & profile & $\mathbf{a}$ \\
\hline 20 & 10 & 6.7 & 5 & 4 & $2 * 10^{-3} / \mathbf{r}$ & $\lambda / 20$ \\
3 & 6 & 9 & 12 & 15 & $15 * 10^{4} \mathrm{r}$ & $\lambda / 20$ \\
5.65 & 8 & 9.76 & 11.52 & 12.64 & $400 \mathrm{r}^{0.5}$ & $\lambda / 20$ \\
15 & 15 & 15 & 15 & 15 & constant & $\lambda / 20$ \\
7 & 9 & 11 & 13 & 15 & Steped & $\lambda / 20$ \\
\hline
\end{tabular}

The scattering widths of the multilayered cylinders are shown in figure 9. Similar to 1D inhomogeneous slab, different profiles of $\varepsilon_{\mathrm{r}}$ can be estimated using several homogeneous layers.

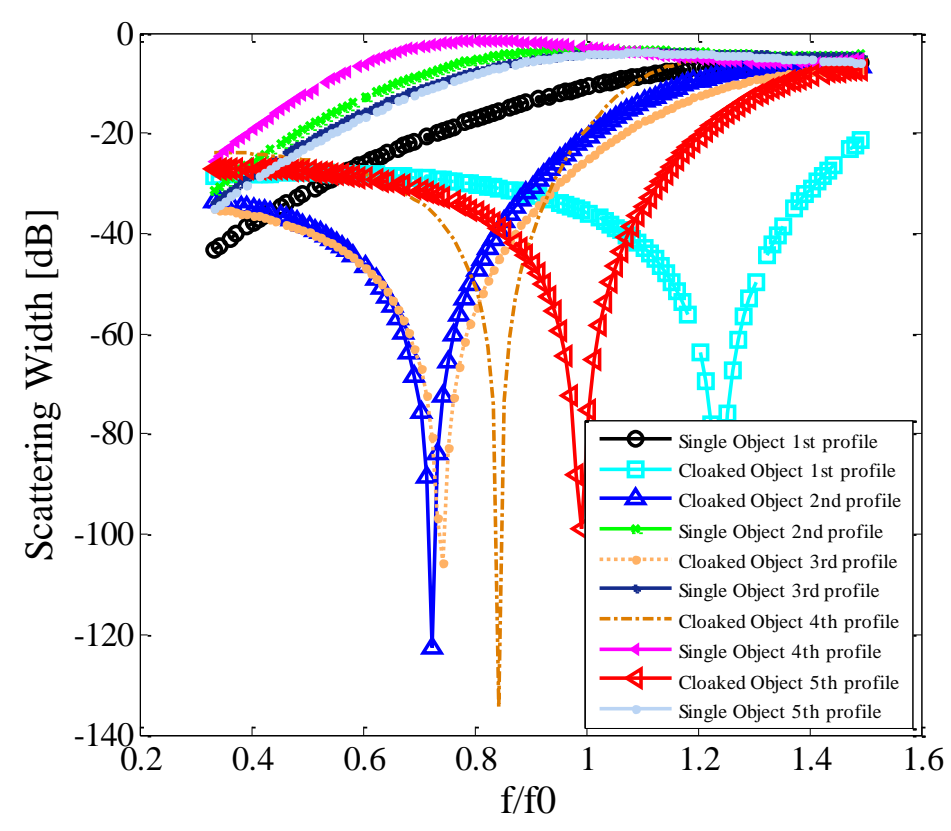

Fig.9. The S parameters for the inhomogeneous cloaked cylinders.

As it is expected, the profiles had smoother variations versus radius have the minimum of scattering width closer to frequency of $\mathrm{f}_{0}$. This means the considered $\mathrm{Z}_{\mathrm{s}}$ for these profiles shows lower error.

\section{IMPLEMENTATION OF NUMERICAL EXAMPLES}

The main purpose of this section is the ability of mantle cloak to make the multilayer structures which are not electrically small invisible. For this reason, some multilayer structures are implemented using CST. The thickness of all structures is chosen equal to $\lambda / 10$. So, the condition of $\beta d<<\lambda_{0}$ is not satisfied and the equations 3 and 4 cannot be used.

The required impedance for cloaking these structures can be obtained with use of FSS surfaces. Here square structure is considered as FSS. This structure is drawn in figure 10-a. Figure 10-b shows whole simulated structure which consists of the object and FSS. Two-layer and five-layer geometries are implemented using CST simulator. The characteristics of both structures and FSSs are given in table IV and V, respectively. 


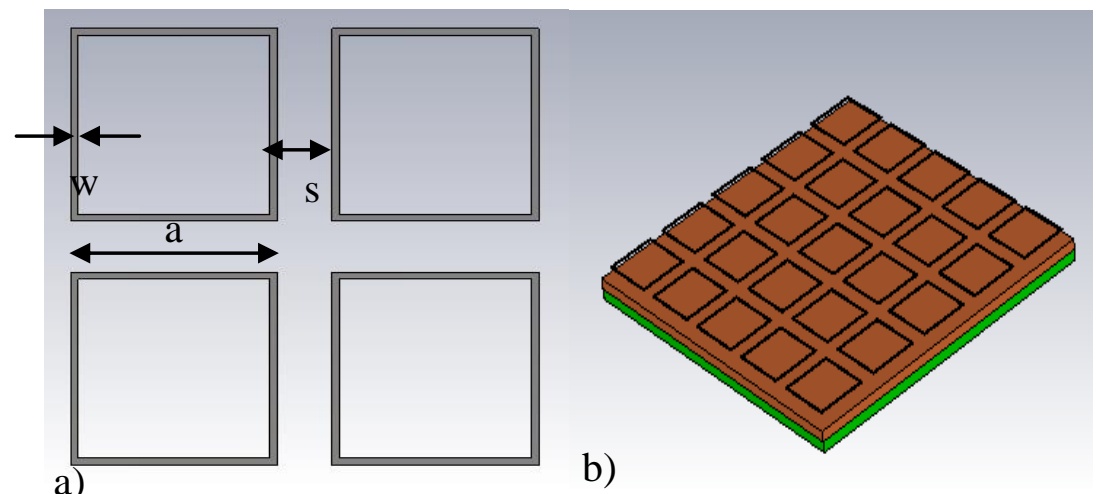

Fig.10. a) The FSS geometry. b) The simulated structure.

TABLE IV. THE DIELECTRIC CONSTANTS OF EACH LAYER FOR BOTH TWO LAYER AND FIVE LAYER STRUCTURES.

\begin{tabular}{llclll}
\hline Dielectric constant & $\boldsymbol{\varepsilon}_{\mathbf{1}}$ & $\boldsymbol{\varepsilon}_{\mathbf{2}}$ & $\boldsymbol{\varepsilon}_{\mathbf{3}}$ & $\boldsymbol{\varepsilon}_{\mathbf{4}}$ & $\boldsymbol{\varepsilon}_{\mathbf{5}}$ \\
\hline Two layer structure & 12 & 8 & - & - & - \\
Five layer structure & 7 & 13 & 10 & 14 & 16 \\
\hline
\end{tabular}

TABLE V. THE PROPERTIES OF THE FSS UTILIZED FOR MAKING THE PROPOSED 2 LAYER AND 5 LAYER STRUCTURES.

\begin{tabular}{llll}
\hline parameters & a & s & d \\
\hline Two layer structure & $\lambda / 5.3$ & $\lambda / 20$ & $\lambda / 30$ \\
Five layer structure & $\lambda / 5$ & $\lambda / 20$ & $\lambda / 30$ \\
\hline
\end{tabular}

a)

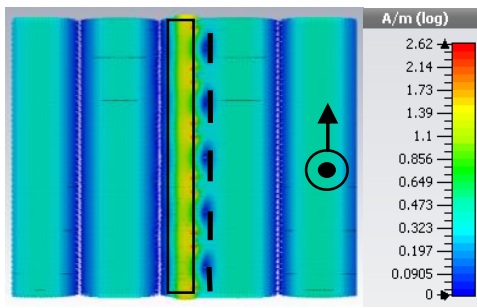

b)

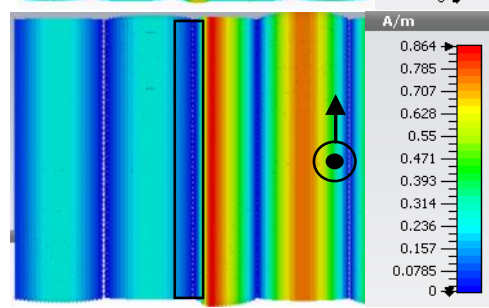

c)

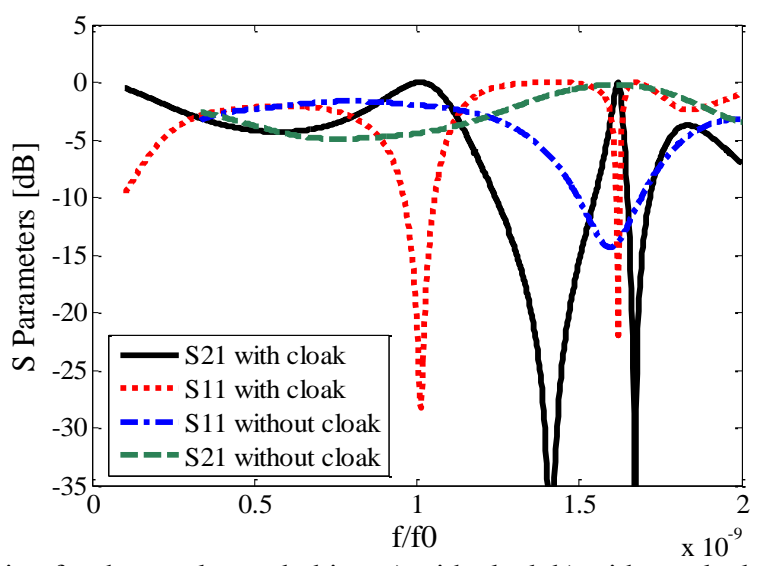

Fig.11. The E field distribution for the two layered object a) with cloak b) without cloak. c) the $\mathrm{S}$ parameters for this structure with and without cloak. 
Where $\mathrm{d}$ is the distance between the FSS and object. The magnitude of reflection and transmission coefficients and also the magnitude of E-field distribution for two layer and 5 layer geometries with and without cloak are shown in figures 11 and 12, respectively.

From figures 11.a and 12.a E field magnitude is not distributed and the scattering already suppressed for cloaked object while E field magnitudes for single dielectrics (figures 11.b and 12.b) are distributed. The S parameters in figures 11.c and 12.c show that the most of the wave transmit so the reflection becomes minimum for both structures at designed frequency. Moreover, these figures indicate S11 has been improved more than $20 \mathrm{~dB}$. It is concluded that the FSS cloak is applicable for multilayer complex structures.

a)

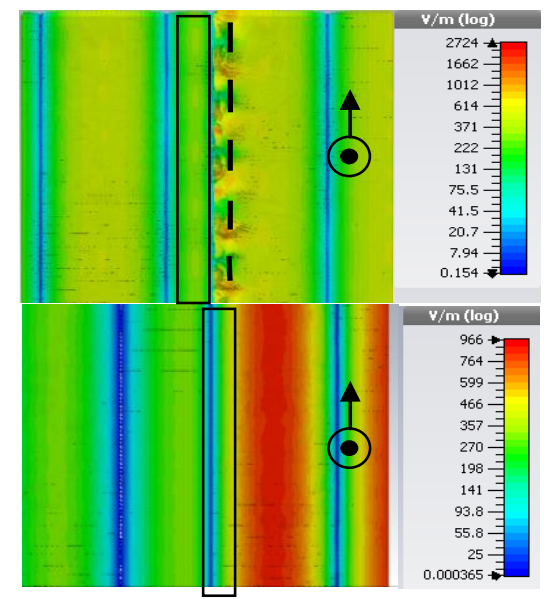

c)

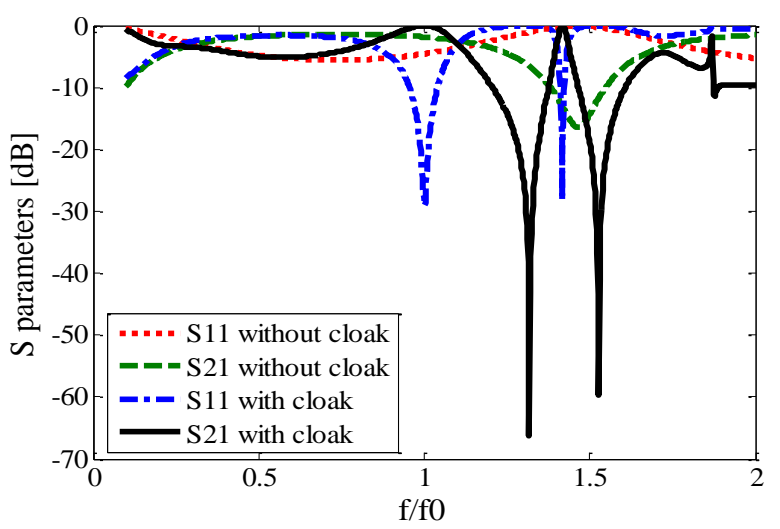

Fig.12. The E field distribution for the 5 layered object a) with cloak b) without cloak. c) the $\mathrm{S}$ parameters for this structure with and without cloak.

\section{CONCLUSION}

An analytical method to calculate the impedance required for cancelation the scattering and make a multilayer structure invisible has been studied. To validate this method some multilayer structures have been considered and suitable performances are achieved. Furthermore, the required covering impedance for making an inhomogeneous slab invisible is computed analytically by dividing the slab into small homogeneous sub-layers. The reflection and transmission coefficients at the design frequency of $f_{0}$ in all structures become minimum and maximum, respectively. In addition, with the use of a full wave simulator some multilayer structures are implemented and simulated. Results show 
no distribution in E field and also a negligible scattering at the design frequency, hence the cloaking is achieved.

\section{REFERENCES}

[1] J. B. Pendry, D. Schurig, and D. R. Smith, "Controlling electromagnetic fields," Science, Vol. 312, pp. 17801782, 2006.

[2] Leonhardt. U, "Optical Conformal Mapping," Science, vol. 312, pp. 1777-1780, 2006.

[3] J. B. Pendry, D. Schurig, and D. R. Smith, "Controlling Electromagnetic Fields," Science 12, pp. 1780-1782, 2006.

[4] Q. Cheng, W. X. Jiang, and T.-J. Cui," Investigations of The Electromagnetic Properties of Three-Dimensional Arbitrarily- Shaped Cloaks", Progress in Electromagnetic Research, pp. 105-117, 2009.

[5] Y. Luo, H. Chen, J. Zhang, L. Ran, and J. A. Kong "Design and Analytically Full-wave Validation of the Invisibility Cloaks, Concentrators, and Field Rotators Created with a General Class of Transformations," Physical Review B, 77-125127, 2007.

[6] J. S. McGuirk, and P. J. Collins, "Controlling the Transmitted Field into a Cylindrical Cloak's Hidden Region", Opt Express, Vol. 16, No. 22, 17560-73, 2008.

[7] N. B. Kundtz, D. R. Smith, and J. B. Pendry, "Electromagnetic Design with Transformation Optics", Proceeding of the IEEE, Vol. 99, No. 10, pp. 1622-1633, 2010

[8] A. Alù, and N. Engheta, "Achieving Transparency with Plasmonic and Metamaterial Coatings," Phys. Rev. E, Vol. 72, 016623, 2005.

[9] A. Alù and N. Engheta, "Cloaking and Transparency for Collections of Particles with Metamaterial and Plasmonic Covers," Optics Express, Vol. 15, 7578, 2007.

[10] A. Alù, "Mantle cloak: invisibility induced by a surface," Phys. Rev. B, Vol. 80, 24115, 2009.

[11] Pai-Yen Chen and Andrea Alu, "Atomically Thin Surface Cloak Using Graphene", ACS Nano, pp.5855-5863, 2011.

[12] A. Monti, L. Tenuti, G. Oliveri, A. Alù, A. Massa, A. Toscano, and F. Bilotti "Design of Multi-Layer Mantle Cloaks," Proceedings of the Eighth International Congress on Advanced Electromagnetic Materials in Microwaves and Optics Metamaterials, pp. 25-29, 2014.

[13] Jason C. Soric, Alessio Monti, Alessandro Toscano, Filiberto Bilotti, and Andrea Alù, "Multiband and Wideband Bilayer Mantle Cloaks" IEEE Transactions on Antennas and Propagation, Vol. 63, pp. 3235-3240, 2015.

[14] J. C. Soric, P. Y. Chen, A. Kerkhoff, D. Rainwater, K. Melin, and A. Alù, "Demonstration of an ultralow profile cloak for scattering suppression of a finite-length rod in free space,” New. J. Phys., Vol. 15, 033037, 2013.

[15] Ensieh Ghasemi Mizuji and Ali Abdolali, "A New Implementation of Frequency Selective Surface Cloak for Cylindrical Structures”, Iranian Journal of Electrical \& Electronic Engineering, Vol. 12, No.2, pp. 91-97, 2016.

[16] Z. H. Jiang, P. E. Sieber, L. Kang, and D. H. Werner, "Restoring Intrinsic Properties of Electromagnetic Radiators Using Ultralight Weight Integrated Meta-surface Cloaks," Adv. Func. Mat., Vol. 25, No. 29, pp. 4708-4716, 2015.

[17] Y. R. Padooru, A. B. Yakovlev, P. Y. Chen, and A. Alù, "Line-Source Excitation of Realistic Conformal Meta-surface Cloaks". J. Appl. Phys., Vol. 112, No. 10, pp. 104902, 2012.

[18] P. Y. Chen, and A. Alù, "Mantle Cloaking Using Thin Patterned Meta-surfaces," Physical Review. B, Vol. 84, 205110, 2011.

[19] Mohamed Farhat, Pai-Yen Chen, S'ebastien Guenneau, Stefan Enoch and Andrea Alu, "Frequency-selective surface acoustic invisibility for three-dimensional immersed objects" Physical Review, Vol. 86, 174303, 2012.

[20] Jason C. Soric, A. Monti, A. Toscano, and F. Bilotti, A. Alù, "Dual-Polarized Reduction of Dipole Antenna Blockage Using Mantle Cloaks", IEEE Transaction on Antennas Propagation, Vol. 63, pp. 4827-4834, 2016.

[21] Monti, J. Soric, M. Barbuto, D. Ramaccia, S. Vellucci, F. Trotta, A. Alù, A. Toscano, and F. Bilotti, "Mantle cloaking for co-site radio-frequency antennas," Appl. Phys. Lett., Vol. 108, No. 11, p. 113502, 2016.

[22] Monti, M. Barbuto, A. Toscano, and F. Bilotti, "Nonlinear Mantle Cloaking Devices for Power-Dependent Antenna Arrays," IEEE Ant. Wirel. Prop. Lett., Vol. 16, pp. 1727 - 1730, 2017.

[23] A. Monti, J. Soric, A. Alù, A. Toscano, and F. Bilotti, "Design of cloaked Yagi-Uda antennas," EPJ Appl. Metamaterials, Vol. 3, p. 10, 2016.

[24] A. Monti, J. Soric, A. Alù, F. Bilotti, A. Toscano, and L. Vegni, "Anisotropic Mantle Cloaks for TM and TE scattering reduction," IEEE Transaction on Antennas Propagation, pp. 1775- 1788, 2015.

[25] Z. H. Jiang and D. H. Werner, "Exploiting Meta-surface Anisotropy for Achieving Near-Perfect Low-Profile Cloaks Beyond The Quasi-Static Limit," J. Phys. D: Appl. Phys., Vol. 46, No. 50, p. 505306, 2013.

[26] F. A. Benson and T. M. Benson, "Fields Waves and Transmission", Springer, pp. 50-82,1991. 
[27] Mahmoud Fallah, Alireza Ghayekhloo and Ali Abdolali," Design of Frequency Selective Band Stop Shield Using Analytical Method", Journal of Microwaves, Optoelectronics and Electromagnetic Applications, Vol. 14, pp. 217-228, December 2015. 